Honam Mathematical J. 36 (2014), No. 1, pp. 167-178

http://dx.doi.org/10.5831/HMJ.2014.36.1.167

\title{
ON DERIVATIONS OF BE-ALGEBRAS
}

\author{
Kyung Ho Kim* and SAng Moon Lee
}

\begin{abstract}
In this paper, we introduce the notion of derivation in a BE-algebra, and consider the properties of derivations. Also, we characterize the fixed set $F i x_{d}(X)$ and Kerd by derivations. Moreover, we prove that if $d$ is a derivation of BE-algebra, every filter $F$ is a $d$-invariant.
\end{abstract}

\section{Introduction}

Y. Imai and K. Iséki introduced two classes of abstract algebras: BCK-algebras and BCI-algebras([3, 4]). It is known that the class of BCK-algebras is a proper subclass of the class of BCI-algebras. In [1, 2], Q. P. Hu and X. Li introduced a wide class of abstracts: $\mathrm{BCH}-$ algebras. They have shown that the class of BCI-algebras is a proper subclass of the class of BCH-algebras. In [5], H. S. Kim and Y. H. Kim introduced the notion of a BE-algebra as a dualization of a generation of a BCK-algebras. In this paper, we introduced the notion of derivation in a BE- algebra, and considered the properties of derivations. Also, we characterized the fixed set Fix $(X)$ and Kerd by derivations. Moreover, we prove that if $d$ is a derivation of BE-algebra, every filter $F$ is a $d$ invariant.

\section{Preliminaries}

In what follows, let $X$ denote an BE-algebra unless otherwise specified.

Received December 26, 2013. Accepted January 9, 2014.

2010 Mathematics Subject Classification. 06F35, 03G25, 08A30.

Key words and phrases. BE-algebra, derivation, self-distributive, filter, normal filter, isotone.

${ }^{*}$ Corresponding author 
By a $B E$-algebra, we mean an algebra $(X ; *, 1)$ of type $(2,0)$ with a single binary operation " $*$ " that satisfies the following identities, for any $x, y, z \in X$,

(BE1) $x * x=1$ for all $x \in X$.

(BE2) $x * 1=1$ for all $x \in X$.

(BE3) $1 * x=x$ for all $x \in X$.

$(\mathrm{BE} 4) x *(y * z)=y *(x * z)$.

A BE-algebra $X$ is said to be self-distributive if $x *(y * z)=(x * y) *$ $(x * z)$ for all $x, y, z \in X$. A non-empty subset $S$ of a BE-algebra $X$ is said to be a subalgebra of $X$ if $x * y \in S$ whenever $x, y \in S$. For any $x, y$ in a BE-algebra $X$, we define $x \vee y=(y * x) * x$. A map $d$ is said to be isotone if $x \leq y$, then $d(x) \leq d(y)$ for all $x, y \in X$.

Let $X$ be a BE-algebra. We define the binary operation " $\leq$ " as the following,

$$
x \leq y \Leftrightarrow x * y=1
$$

for all $x, y \in X$.

Let $X$ be a BE-algebra. Then the following identities are true for all $x, y, z \in X$.

(p1) $x *(y * x)=1$.

(p2) $x *((x * y) * y))=1$.

(p3) Let $X$ be a self-distributive BE-algebra. If $x \leq y$, then $z * x \leq z * y$ and $y * z \leq x * z$.

Let $X$ be a BE-algebra and let $F$ be a non-empty subset of $X$. Then $F$ is called a filter of $X$ if

(F1) $1 \in F$,

(F2) If $x \in F$ and $x * y \in F$, then $y \in F$.

\section{Derivations of BE-algebras}

Definition 3.1. A self-map $d$ on a BE-algebra $X$ is called a derivation if

$$
d(x * y)=(x * d(y)) \vee(d(x) * y)
$$

for every $x, y \in X$.

Example 3.2. Let $X=\{1, a, b\}$ be a set in which "*" is defined by 


\begin{tabular}{c|ccc}
$*$ & 1 & $a$ & $b$ \\
\hline 1 & 1 & $a$ & $b$ \\
$a$ & 1 & 1 & 1 \\
$b$ & 1 & 1 & 1
\end{tabular}

Then $X$ is a BE-algebra. Define a map $d: X \rightarrow X$ by

$$
d(x)= \begin{cases}1 & \text { if } x=1 \\ b & \text { if } x=a \\ a & \text { if } x=b\end{cases}
$$

Then it is easy to check that $d$ is a derivation of $X$.

Example 3.3. Let $X=\{1, a, b, c\}$ be a set in which "*" is defined by

\begin{tabular}{c|cccc}
$*$ & 1 & $a$ & $b$ & $c$ \\
\hline 1 & 1 & $a$ & $b$ & $c$ \\
$a$ & 1 & 1 & $a$ & $a$ \\
$b$ & 1 & 1 & 1 & $a$ \\
$c$ & 1 & 1 & $a$ & 1
\end{tabular}

Then $X$ is a BE-algebra. Define a map $d: X \rightarrow X$ by

$$
d(x)= \begin{cases}1 & \text { if } x=1, a \\ a & \text { if } x=b, c\end{cases}
$$

Then it is easy to check that $d$ is a derivation of a BE-algebra $X$.

Example 3.4. Let $X=\{1, a, b, c\}$ be a set in which "*" is defined by

\begin{tabular}{c|cccc}
$*$ & 1 & $a$ & $b$ & $c$ \\
\hline 1 & 1 & $a$ & $b$ & $c$ \\
$a$ & 1 & 1 & $b$ & 1 \\
$b$ & 1 & $c$ & 1 & $c$ \\
$c$ & 1 & 1 & $b$ & 1
\end{tabular}

Then $X$ is a BE-algebra. Define a map $d: X \rightarrow X$ by

$$
d(x)= \begin{cases}1 & \text { if } x=1, b \\ c & \text { if } x=a, c .\end{cases}
$$

Then it is easy to check that $d$ is a derivation of $X$.

Example 3.5. Let $X=\{1, a, b, c\}$ be a set in which "*" is defined by 


\begin{tabular}{c|cccc}
$*$ & 1 & $a$ & $b$ & $c$ \\
\hline 1 & 1 & $a$ & $b$ & $c$ \\
$a$ & 1 & 1 & $b$ & $c$ \\
$b$ & 1 & $a$ & 1 & $c$ \\
$c$ & 1 & 1 & 1 & 1
\end{tabular}

Then $X$ is a BE-algebra. Define a map $d: X \rightarrow X$ by

$$
d(x)= \begin{cases}1 & \text { if } x=1, b, c \\ a & \text { if } x=a\end{cases}
$$

Then it is easy to check that $d$ is a derivation of $X$.

Proposition 3.6. Let $d$ be a derivation of $X$. Then we have

(1) $d(1)=1$,

(2) $d(x)=d(x) \vee x$ for all $x \in X$.

Proof. (1) Let $d$ be a derivation of $X$. Then we have

$$
\begin{aligned}
d(1) & =d(1 * 1)=(1 * d(1)) \vee(d(1) * 1) \\
& =d(1) \vee 1=(1 * d(1)) * d(1)=d(1) * d(1)=1 .
\end{aligned}
$$

(2) For all $x \in X$, we have

$$
\begin{aligned}
d(x) & =d(1 * x)=(1 * d(x)) \vee(d(1) * x) \\
& =d(x) \vee(1 * x)=d(x) \vee x .
\end{aligned}
$$

Proposition 3.7. Let $d$ be a derivation of $X$. Then the following identities hold:

(1) $x \leq d(x)$ for all $x \in X$,

(2) $d(x) * y \leq x * y \leq x * d(y)$ for all $x, y \in X$.

Proof. (1) By Proposition 3.6(2) and (BE4), we have for all $x \in X$,

$$
\begin{aligned}
x * d(x) & =x *(d(x) \vee x)=x *((x * d(x)) * d(x)) \\
& =(x * d(x)) *(x * d(x))=1
\end{aligned}
$$

which implies $x \leq d(x)$.

(2) By (1) and (p3), we have $d(x) * y \leq x * y \leq x * d(y)$ for all $x, y \in X$.

Theorem 3.8. Let $X$ be a self-distributive $B E$-algebra and let $d$ be a derivation of $X$. Then we have $d(x * y)=x * d(y)$ for all $x, y \in X$. 
Proof. Let $d$ be a derivation on $X$ and $x, y \in X$. Then we have $d(x) *$ $y \leq x * d(y)$ from Proposition $3.7(2)$. Hence we get

$$
\begin{aligned}
d(x * y) & =(x * d(y)) \vee(d(x) * y) \\
& =((d(x) * y) *(x * d(y))) *(x * d(y)) \\
& =1 *(x * d(y))=x * d(y) .
\end{aligned}
$$

Proposition 3.9. If $d$ is a derivation of $X$, then we have $d(x * d(x))=$ $d(d(x) * x)=1$ for all $x \in X$.

Proof. Let $d$ be a derivation of $X$. Then we have

$$
\begin{aligned}
d(x * d(x)) & =(x * d(d(x))) \vee(d(x) * d(x)) \\
& =(x * d(d(x))) \vee 1=1
\end{aligned}
$$

for all $x \in X$. Similarly, we get

$$
\begin{aligned}
d(d(x) * x) & =(d(x) * d(x)) \vee(d(d(x))) * x) \\
& =1 \vee(d(d(x))) * x)=1
\end{aligned}
$$

for all $x \in X$.

Proposition 3.10. Let $X$ be a BE-algebra. Then

$$
x \leq d_{n}\left(d_{n-1}\left(\ldots\left(d_{2}\left(d_{1}(x)\right)\right) \ldots\right)\right)
$$

for $n \in \mathbb{N}$, where $d_{1}, d_{2}, \ldots, d_{n}$ are derivations of $X$.

Proof. For $n=1$,

$$
\begin{aligned}
d_{1}(x) & =d_{1}(1 * x)=\left(1 * d_{1}(x)\right) \vee\left(d_{1}(1) * x\right)=d_{1}(x) \vee(1 * x) \\
& =d_{1}(x) \vee x=\left(x * d_{1}(x)\right) * d_{1}(x) .
\end{aligned}
$$

Hence we have

$$
x * d_{1}(x)=x *\left(\left(x * d_{1}(x)\right) * d_{1}(x)\right)=\left(x * d_{1}(x)\right) *\left(x * d_{1}(x)\right)=1
$$

which implies $x * d_{1}(x)=1$. Thus $x \leq d_{1}(x)$.

Let $n \in \mathbb{N}$ and $x \leq d_{n}\left(d_{n-1}\left(\ldots\left(d_{2}\left(d_{1}(x)\right)\right) \ldots\right)\right)$. For simplicity, let

$$
D_{n}=d_{n}\left(d_{n-1}\left(\ldots\left(d_{2}\left(d_{1}(x)\right)\right) \ldots\right)\right) \text {. }
$$

Then

$$
\begin{aligned}
d_{n+1}\left(D_{n}\right) & =d_{n+1}\left(1 * D_{n}\right)=\left(1 * d_{n+1}\left(D_{n}\right)\right) \wedge\left(d_{n+1}(1) * D_{n}\right) \\
& =d_{n+1}\left(D_{n}\right) \wedge D_{n}=\left(D_{n} * d_{n+1}\left(D_{n}\right)\right) * d_{n+1}\left(D_{n}\right) .
\end{aligned}
$$

Hence $D_{n} * D_{n+1}=1$, which implies $D_{n} \leq D_{n+1}$. By assumption, $x \leq$ $D_{n} \leq D_{n+1}$. 
Let $d$ be a derivation of $X$. Define a set $F i x_{d}(X)$ by

$$
F_{i x}(X):=\{x \in X \mid d(x)=x\}
$$

for all $x \in X$.

Proposition 3.11. Let $d$ be a derivation of $X$. Then $F i x_{d}(X)$ is a subalgebra of $X$.

Proof. Clearly, $1 \in F_{i x}(X)$ and so $F i x_{d}(X)$ is nonempty. Let $x, y \in$ $F i x_{d}(X)$. Then we have $d(x)=x$ and $d(y)=y$, and so

$$
d(x * y)=(x * d(y)) \vee(d(x) * y)=(x * y) \vee(x * y)=x * y .
$$

This implies $x * y \in F i x_{d}(X)$. Hence $F_{i x}(X)$ is a subalgebra of $X$.

Proposition 3.12. If $x, y \in F i x_{d}(X)$, then $x \vee y \in F i x_{d}(X)$.

Proof. Let $x, y \in F i x_{d}(X)$. Then we get

$$
\begin{aligned}
d(x \vee y) & =d((y * x) * x) \\
& =((y * x) * d(x)) \vee(d(y * x) * y) \\
& =((y * x) * x) \vee((y * d(x) \vee d(y) * x) * x) \\
& =((y * x) * x) \vee((y * x) * x) \\
& =(y * x) * x=x \vee y .
\end{aligned}
$$

Proposition 3.13. Let $d$ be a derivation of $X$. If $x \in F i x_{d}(X)$, then we have $(d \circ d)(x)=x$.

Proof. Let $x \in F i x_{d}(X)$. Then we have

$$
(d \circ d)(x)=d(d(x))=d(x)=x .
$$

This completes the proof.

Proposition 3.14. Let $d$ be a derivation of a self-distributive BEalgebra $X$. If $y \in F i x_{d}(X)$, then we have $x * y \in F i x_{d}(X)$ for all $x \in X$.

Proof. Let $y \in F_{i x}(X)$. Then we have $d(y)=y$. Hence we have

$$
\begin{aligned}
d(x * y) & =(x * d(y)) \vee(d(x) * y)=((d(x) * y) *(x * y)) *(x * y) \\
& =(x *((d(x) * y) * y)) *(x * y) \\
& =((x *(d(x) * y)) *(x * y)) *(x * y) \\
& =((x * d(x)) *(x * y)) *(x * y)) *(x * y) \\
& =((1 *(x * y)) *(x * y)) *(x * y) \\
& =((x * y) *(x * y)) *(x * y)=1 *(x * y)=x * y .
\end{aligned}
$$

This completes the proof. 
Theorem 3.15. Let $X$ be a $B E$-algebra and let $d_{1}$ and $d_{2}$ be two isotone derivations on $X$. If $d(x) \in F i x_{d}(X)$, then $d_{1}=d_{2}$ if and only if $F i x_{d_{1}}(X)=F_{i x_{d_{2}}}(X)$.

Proof. Let $d_{1}=d_{2}$. Then $F i x_{d_{1}}(X)=F i x_{d_{2}}(X)$. Conversely, let $F_{i x_{d_{1}}}(X)=F_{i x_{d_{2}}}(X)$ and $d(x) \in F i x_{d}(X)$ for $x \in X$. Then $d^{2}=d$. Thus $d_{1}(x) \in F i x_{d_{1}}(X)=F i x_{d_{2}}(X)$ since $d_{1}\left(d_{1}(x)\right)=d_{1}^{2}(x)=d_{1}(x)$. Hence $d_{2}\left(d_{1}(x)\right)=d_{1}(x)$. Also, $d_{2}(x) \in F i x_{d_{2}}(X)=F i x_{d_{1}}(X)$, and so $d_{1}\left(d_{2}(x)\right)=d_{2}(x)$. Since $x \leq d_{1}(x)$, we have $d_{1}\left(d_{2}(x)\right)=d_{2}(x) \leq$ $d_{2}\left({ }_{1}(x)\right)$. Symmetrically, we have $d_{1}\left(d_{2}(x)\right) \leq d_{2}\left(d_{1}(x)\right)$. Hence $d_{1} d_{2}=$ $d_{2} d_{1}$. It follows that $d_{2}(x)=d_{1}\left(d_{2}(x)\right)=d_{2}\left(d_{1}(x)\right)=d_{1}(x)$.

Definition 3.16. Let $X$ be a BE-algebra and let $d$ be a derivation of $X$.

(1) $d$ is called a monomorphic derivation if $d$ is one-to- one.

(2) $d$ is called an epic derivation if $d$ is onto.

Theorem 3.17. Let $d$ be a derivation of $X$ and $d(x) \in F i x_{d}(X)$. Then the following conditions are equivalent:

(1) $d(x)=x$ for all $x \in X$,

(2) $d$ is a monomorphic derivation,

(3) $d$ is an epic derivation.

Proof. (1) $\Rightarrow(2)$ is clear.

$(2) \Rightarrow(1)$ Let $d$ be a monomorphic derivation and $x \in X$. Since $d(x) \in$ Fix $_{d}(X)$, we have $d(d(x))=d(x)$. Since $d$ is monomorphic, $d(x)=x$ for all $x \in X$.

(1) $\Rightarrow(3)$ is trivial.

$(3) \Rightarrow(1)$ Let $d$ be an epic derivation and $x \in X$. Then there exists $y \in X$ such that $d(y)=x$. Since $d(x) \in F i x_{d}(X)$, we have $d(x)=d(d(y))=$ $d^{2}(y)=d(y)=x$.

Let $d$ be a derivation of $X$. Define a Kerd by

$$
\operatorname{Kerd}=\{x \mid d(x)=1\}
$$

for all $x \in X$.

Proposition 3.18. Let $d$ be a derivation of $X$. Then Kerd is a subalgebra of $X$.

Proof. Clearly, $1 \in K e r d$, and so Kerd is non-empty. Let $x, y \in$ $K e r d$. Then $d(x)=1$ and $d(y)=1$. Hence we have

$$
d(x * y)=x * d(y) \vee d(x) * y=(x * 1) \vee(1 * y)=1 \vee y=1,
$$


and so $x * y \in$ Kerd. Thus Kerd is a subalgebra of $X$.

A BE-algebra $X$ is said to be commutative if for all $x, y \in X$,

$$
(y * x) * x=(x * y) * y .
$$

Proposition 3.19. Let $X$ be a commutative BE-algebra. If $x \in$ Kerd and $x \leq y$, then we have $y \in K e r d$.

Proof. Let $x \in$ Kerd and $x \leq y$. Then $d(x)=1$ and $x * y=1$.

$$
\begin{aligned}
d(y) & =d(1 * y)=d((x * y) * y)=d((y * x) * x) \\
& =((y * x) * d(x)) \vee(d(y * x) * x) \\
& =((y * x) * 1) \vee(d(y * x) * x) \\
& =1 \vee(d(y * x) * x)=1,
\end{aligned}
$$

and so $y \in K e r d$. This completes the proof.

Theorem 3.20. Let $d$ be an isotone derivation of a self-distributive $B E$-algebra $X$. If $d$ is idempotent, then Kerd is a filter of $X$.

Proof. Clearly, $1 \in$ Kerd. Let $x \in \operatorname{Kerd}$ and $x * y \in$ Kerd. Then we have $1=d(x * y)=x * d(y)$ from Theorem 3.8, which means $x \leq d(y)$. Hence we get $1=d(x) \leq d^{2}(y)=d(y)$, i.e., $d(y)=1$. This implies $y \in$ Kerd. This completes the proof.

Proposition 3.21. Let $d$ be a derivation of $X$ and $x \in$ Kerd. Then we have $x \vee y \in$ Kerd for all $y \in X$.

Proof. Let $d$ be a derivation of $X$ and $x \in \operatorname{Kerd}$. Then $d(x)=1$. Hence we have for all $y \in X$,

$$
\begin{aligned}
d(x \vee y) & =d((y * x) * x)=((y * x) * d(x)) \vee(d(y * x) * x) \\
& =((y * x) * 1) \vee(d(y * x) * x)=1 \vee(d(y * x) * x)=1 .
\end{aligned}
$$

Hence we have $x \vee y \in$ Kerd.

Proposition 3.22. Let $d$ be a derivation of $X$ and $y \in K e r d$. Then we have $x * y \in$ Kerd for all $x \in X$.

Proof. Let $d$ be a derivation of $X$ and $y \in \operatorname{Kerd}$. Then $d(y)=1$. Hence we have for all $x \in X$,

$$
\begin{aligned}
d(x * y) & =(x * d(y)) \vee(d(x) * y) \\
& =(x * 1) \vee(d(x) * y)=1 \vee d(x) * y)=1 .
\end{aligned}
$$

Proposition 3.23. Let $d$ be a derivation of $X$. If $d$ is one-to-one, then $\operatorname{Kerd}=1$. 
Proof. Suppose that $d$ is one-to-one and $x \in \operatorname{Kerd}$. Then $d(x)=1=$ $d(1)$, and thus $x=1$, i.e., $\operatorname{Kerd}=\{1\}$.

Definition 3.24. Let $X$ be a BE-algebra. A self-map $d$ is isotone if $x \leq y$ implies $d(x) \leq d(y)$.

Proposition 3.25. Let $d$ be a derivation of a BE-algebra $X$. If $d(x) \vee$ $d(y) \leq d(x \vee y)$ for all $x, y \in X$, then $d$ is an isotone derivation of $X$.

Proof. Suppose that $d(x) \vee d(y) \leq d(x \vee y)$ and $x \leq y$. Then we have $d(x) \leq d(x) \vee d(y) \leq d(x \vee y)=d(y)$.

Proposition 3.26. Let $d$ be an isotone derivation of $X$. If $x \leq y$ and $x \in$ Kerd, then $y \in$ Kerd.

Proof. Let $x \leq y$ and $x \in \operatorname{Kerd}$. Then we have $d(x)=1$, and so

$$
1=d(x) \leq d(y)
$$

which implies $d(y)=1$.

Definition 3.27. Let $X$ be a BE-algebra. A non-empty subset $F$ of $X$ is said to be a $d$-invariant if $d(F) \subseteq F$ where $d(F)=\{d(x) \mid x \in F\}$.

Proposition 3.28. Let $X$ be a BE-algebra and let $d$ be a derivation. Then every filter $F$ is a $d$-invariant.

Proof. Let $F$ be a filter of $X$. Let $y \in d(F)$. Then $y=d(x)$ for some $x \in F$. It follows that $x * y=x * d(x)=1 \in F$, which implies $y \in F$. Thus $d(F) \subseteq F$. Hence $F$ is a $d$-invariant.

Let $X$ be a BE-algebra. We define the binary operation " + " as the following

$$
x+y=(x * y) * y
$$

for all $x, y \in X$. Clearly, $X$ is a commutative BE-algebra if and only if $x+y=y+x$ for all $x, y \in X$.

Proposition 3.29. Let $X$ be a commutative BE-algebra. Then the followings hold for all $x, y, z \in X$,

(1) $x+1=1+x$.

(2) $x+y=y+x$.

(3) $x+(y+z)=(x+y)+z$.

(4) $x+x=x$.

Proof. The proof is straightforward.

Proposition 3.30. Let $X$ be a $B E$-algebra and let $d$ be a derivation of $X$. Then the followings hold for all $x, y \in X$, 
(1) $d(x+1)=1$.

(2) $d(x+x)=d(x)+x$.

(3) $d(x)=x+d(x)$.

Proof. (1) Let $x \in X$. Then we get

$$
\begin{aligned}
d(x+1) & =d((x * 1) * 1)=((x * 1) * d(1)) \vee(d(x * 1) * 1) \\
& =(1 * 1) \vee(d(x * 1) * 1)=1 \vee 1 \\
& =1
\end{aligned}
$$

(2) Let $x \in X$. Then we have

$$
\begin{aligned}
d(x+x) & =d((x * x) * x)=((x * x) * d(x)) \vee(d(x * x) * x) \\
& =(1 * d(x)) \vee(d(1) * x)=d(x) \vee x \\
& =(x * d(x)) * d(x)=x+d(x)
\end{aligned}
$$

(3) Let $x \in X$. Then we have

$$
\begin{aligned}
d(x) & =d(1 * x)=(1 * d(x)) \vee(d(1) * x) \\
& =d(x) \vee x=(x * d(x)) * d(x) \\
& =x+d(x) .
\end{aligned}
$$

Definition 3.31. Let $X$ be a BE-algebra. A non-empty set $F$ of $X$ is called a normal filter of $X$ if it satisfies the following conditions:

(NF1) $1 \in F$,

(NF2) $x \in X$ and $y \in F$ imply $x * y \in F$. by

Example 3.32. Let $X=\{1, a, b, c\}$ be a set in which "*" is defined

\begin{tabular}{c|cccc}
$*$ & 1 & $a$ & $b$ & $c$ \\
\hline 1 & 1 & $a$ & $b$ & $c$ \\
$a$ & 1 & 1 & $b$ & $c$ \\
$b$ & 1 & 1 & 1 & $c$ \\
$c$ & 1 & 1 & 1 & 1
\end{tabular}

Then $X$ is a BE-algebra. Let $F=\{1, a\}$. Then $F$ is a normal filter of $X$.

Proposition 3.33. Let $X$ be a self-distributive BE-algebra and let $d$ be a derivation of $X$. Then Fix $(X)$ is a normal filter of $X$.

Proof. Clearly, $1 \in F_{i x}(X)$. Let $x \in X$ and $y \in F i x_{d}(X)$. Then we have $d(y)=y$, and so

$$
\begin{aligned}
d(x * y) & =x * d(y) \\
& =x * y,
\end{aligned}
$$


which implies $x * y \in F i x_{d}(X)$ from Theorem 3.8. This completes the proof.

Proposition 3.34. Let $X$ be a self-distributive BE-algebra and let $d$ be a derivation of $X$. Then Kerd is a normal filter of $X$.

Proof. Clearly, $1 \in$ Kerd. Let $x \in X$ and $y \in K e r d$. Then we have $d(y)=1$, and so from Theorem 3.8 ,

$$
d(x * y)=x * d(y)=x * 1=1,
$$

which implies $x * y \in K e r d$. Hence Kerd is a normal filter of $X$.

Proposition 3.35. Let $X$ be a self-distributive $B E$-algebra and let $d$ be a derivation of $X$. Then $F_{a}=\{x \in X \mid a \leq d(x)\}$ is a normal filter of $X$.

Proof. Clearly, $a \leq d(1)=1$ for any $a \in X$, and so $1 \in F_{a}$. Let $x \in X$ and $y \in F_{a}$. Then we have $a * d(y)=1$, and so from Theorem 3.8,

$$
\begin{aligned}
a * d(x * y) & =a *(x * d(y))=(a * x) *(a * d(y)) \\
& =(a * x) * 1 \\
& =1
\end{aligned}
$$

which implies $x * y \in F_{a}$. Hence $F_{a}$ is a normal filter of $X$.

\section{References}

[1] Q. P. Hu and X. Li, On BCH-algebras, Math. Seminar Notes 11 (1983), 313-320.

[2] Q. P. Hu and X. Li, On proper BCH-algebras, Math Japonicae 30 (1985), 659661.

[3] K. Iseki and S. Tanaka, An introduction to theory of BCK-algebras, Math Japonicae 23 (1978), 1-20.

[4] K. Iseki, On BCI-algebras, Math. Seminar Notes 8 (1980), 125-130.

[5] H. S. Kim and Y. H. Kim, On BE-algebras, Sci. Math. Japo. 66(1) (2007), 113-116.

[6] S. S. Ahn and K. S. So, On ideals and uppers in BE-algebras, Sci. Math. Japo. Online e-2008, 351-357.

Kyung Ho Kim

Department of Mathematics,

Korea National University of transportation,

Chungju 380-702, Korea. 
E-mail : ghkim@ut.ac.kr

Sang Moon Lee

Department of Computer Sciences,

Korea National University of Transportation,

Chungju 380-702, Korea.

E-mail : smlee@ut.ac.kr 